\title{
Teletrabajo en España, acuerdo marco y administración pública
}

\author{
Ángel Belzunegui Eraso \\ Universidad Rovira i Virgili \\ angel.belzunegui@urv.cat
}

\section{Resumen}

En este artículo se presenta la evolución del teletrabajo en los países europeos y en España en los últimos años, en cuanto a la magnitud de su presencia como a los sectores y actividades que se han visto más proclives a implantarlo. Por otro lado se realiza un estado de la cuestión acerca de la regulación del teletrabajo en el entorno de la UE, en términos comparativos, a partir del Acuerdo Marco Europeo de Teletrabajo del 2002 y se informa sobre las estrategias que los actores sociales y la Administración han tomado para paliar la falta de normatividad en la regulación de las prácticas asociadas al teletrabajo.

Palabras clave: teletrabajo; actores sociales; administración pública.

\begin{abstract}
This article discusses the evolution of telework in European countries and in Spain in recent years in terms of the extent to which it has been implemented and which sectors and activities have been most affected. It also presents the state of the art of the regulation of telework in the context of the EU since the European Framework Agreement on Telework in 2002 and informs about the strategies that social actors and the Administration have adopted to mitigate the lack of of regulation of the practices associated with telework.
\end{abstract}

Key words: telework; social agents; public administration 


\section{Introducción: algunas consideraciones previas}

En las dos últimas décadas el teletrabajo ha sido presentado, con mayor o menor intensidad mediática, como una «nueva forma de trabajo», forma que se apartaría del «clásico» trabajo en el domicilio recogido en el Estatuto de los Trabajadores. La novedad de esta forma de trabajar puede venir refrendada por la polisemia del propio concepto teletrabajo; con este término se han querido significar diferentes formas de prestación (existencia o no de laboralidad, por ejemplo), diferentes lugares del ejercicio de la prestación (domicilio, centro de recursos compartidos, teletrabajo itinerante...), diferentes modalidades del ejercicio (on line, off line...) y diferentes instrumentos tecnológicos para realizar la prestación (hardware, software, soporte telemático...). A estas peculiaridades distintivas podrían añadirse, también, todas aquellas que tienen que ver con las condiciones de trabajo como marco en las que se desarrolla la prestación, condiciones que, según los escasos estudios centrados en las mismas, apuntan a una vasta heterogeneidad y discrecionalidad a la hora de diseñar lo que se denomina teletrabajo.

En España no se ha desarrollado una definición diferente de la que se deriva del Acuerdo Marco Europeo sobre Teletrabajo ${ }^{1}$. En las diferentes definiciones sobre teletrabajo encontradas en la bibliografía o en estudios especializados se acentúan las siguientes características: (1) es un tipo de prestación que utiliza las tecnologías de la información y la comunicación on line con el empleador y/o con el cliente, y (2) se realiza desde lugares remotos o alejados de la empresa u organización con la que se tiene vínculos contractuales. Es importante señalar que esta definición no se circunscribe necesariamente a una vinculación contractual de laboralidad con la organización para la que se presta el servicio, de tal manera que incluye también al teletrabajo autónomo. A pesar de ello, el Acuerdo Marco Europeo sobre Teletrabajo (en adelante, AMET) cuando habla de teletrabajo se refiere a aquel tipo de prestación que supone una cierta relación laboral, en una dirección, desde nuestro punto de vista, englobante de diversas formas de trabajo, incluida la que realizan los trabajadores autónomos muy presentes en ciertas actividades que se desarrollan bajo las formas del teletrabajo.

Tampoco existe por parte de los interlocutores sociales (organizaciones empresariales y organizaciones sindicales) ni de las instancias administrativas una definición consensuada de lo que es teletrabajo ni de de las condiciones laborales a las que ha de

\footnotetext{
1 Acuerdo firmado el 16 de julio de 2002, en Bruselas, por los interlocutores sociales europeos CES (Confederación Europea de Sindicatos), UNICE/UEAPME (Unión de Confederaciones de la Industria y de Empresarios de Europa, ahora Bussines Europe), la Unión Europea del Artesanado y de la Pequeña y Mediana Empresa y el CEEP (Centro Europeo de la Empresa Pública), incluyendo representantes del comité de enlace CEC/Eurocuadros. Los firmantes consideraron que el teletrabajo puede servir al objetivo de modernización de las empresas y organizaciones de servicios públicos, así como medio para que los trabajadores concilien vida profesional y vida personal y gocen de una mayor autonomía en el cumplimiento de sus tareas.
} 
estar sujeto. Los sindicatos y las organizaciones empresariales españolas se atienen, en principio, a la definición expresada en el Acuerdo Marco pero sin dotarle de ninguna efectividad jurídica ni regulatoria. No hay que olvidar que el AMET se firma con el mínimo compromiso por lo que se refiere a los contenidos que dan forma a las principales problemáticas del teletrabajo, como, por ejemplo, la jornada, el control del tiempo de trabajo, la carga de trabajo y los derechos sindicales, aunque, es cierto que el AMET sugiere que los teletrabajadores estén sujetos a los títulos que dan cobertura al resto de trabajadores en los aspectos señalados. Por otro lado, también se reconoce en el AMET la laboralidad «especial» que se deriva de esta «nueva forma de trabajo» $y$, en consecuencia, se produce una situación proclive a la libre interpretación y/o a la discrecionalidad a la hora de implementar las prácticas relacionadas con el teletrabajo. El hecho de que los interlocutores sociales europeos no hayan llegado a concretar más los aspectos centrales de las problemáticas asociadas al teletrabajo, indica, una vez más, las dificultades para el establecimiento de acuerdos consistentes que regulen ciertos ámbitos de prestación laboral que nacen, precisamente, al albur de la desregulación de las relaciones laborales y del mercado de trabajo. En resumen, el AMET se apoyó en una serie de principios generales: 1) la naturaleza voluntaria del teletrabajo y del retorno al trabajo presencial en instalaciones de la empresa, y 2) la igualdad de derechos y de tratamiento de los teletrabajadores en relación al resto de la plantilla, haciendo hincapié en los derechos de representación colectiva, seguridad y salud en el trabajo, formación y puesta a disposición de los equipos de trabajo necesarios.

Ante la dificultad que plantea la consideración de lo que jurídicamente ha de ser teletrabajo, los interlocutores sociales en España vienen considerando que existe teletrabajo cuando los sujetos de la prestación realizan todo o parte de su trabajo utilizando tecnologías de la información y telecomunicaciones, englobando cuatro formas de teletrabajo: (1) el realizado en casa de manera puntual, (2) el trabajo móvil (durante viajes o en las casas de los clientes), (3) el denominado SOHO (small office, home office), es decir, en que el domicilio propio es la principal base del trabajo, y (4) aquél que se realiza desde instalaciones compartidas y externas a las empresas o al domicilio, como los centros de recursos compartidos, los telecentros o los telecottages.

La definición institucional más reciente en España es la aportada por la Orden ministerial APU/1981/2006, de 21 de junio, que promueve la implantación de programas piloto de teletrabajo en los departamentos del Ministerio de Administraciones públicas. Esta ha sido la primera norma alineada con el Acuerdo Marco Europeo sobre Teletrabajo. Esta Orden ministerial define el teletrabajo como «modalidad de prestación de servicios de carácter no presencial en virtud de la cual un empleado de la Administración General del Estado puede desarrollar parte de su jornada laboral mediante el uso de medios telemáticos desde su propio domicilio, siempre que las ne- 
cesidades del servicio lo permitan y en el marco de la política de conciliación de la vida personal y familiar y laboral de los empleados públicos» ${ }^{2}$. A continuación se define la figura del teletrabajador (en el ámbito del ejercicio de la Administración del Estado) como «aquel empleado o empleada pública que, en el desempeño de su puesto de trabajo, alterna su presencia en el centro de trabajo en el que desarrolle sus funciones con alguna de las modalidades de jornada de carácter no presencial establecidas en los programas de teletrabajo aprobados anualmente por los departamentos ministeriales o sus organismos públicos».

\section{Evolución del teletrabajo}

Según el informe Status Report on European Telework. New Methodos of Work 1999, publicado por la Dirección General de la Sociedad de la Información de la Comisión Europea, los datos estimados del teletrabajo en España eran los siguientes:

- En 1999 había 162.000 personas que teletrabajaban en su domicilio un día a la semana como mínimo, a jornada completa y por cuenta ajena. Esta cifra representaba, entonces, el 1,3\% sobre el total de la fuerza de trabajo.

- Los teletrabajadores móviles que realizaban al menos 10 horas a la semana fuera del lugar tradicional de trabajo eran aproximadamente 32.000 , lo que representaba un $0,5 \%$ de la fuerza de trabajo.

Por último, el citado informe cuantificaba el número de teletrabajadores autónomos en 32.000, representando el 0,3\% del total de trabajadores.

Según un informe presentado en la IX Asamblea Europea del Teletrabajo (París, 25-27 de septiembre de 2002), En España la cifra de asalariados que trabajaba desde casa al menos un día a la semana, habría aumentado desde 1999: del 1,3\% a cerca del 4,9\%. También otras modalidades de e-trabajo, como el trabajo móvil y los ciberautónomos habrían crecido: de ser prácticamente inexistentes en 1999 pasarían a representar en 2002 cerca del 3\% de la población activa

Sin embargo estos datos han de ser tomados con prudencia. En España no existe un registro estadístico de la incidencia del teletrabajo, por lo tanto es arriesgado dar cifras sobre el peso porcentual del mismo respecto al total de la fuerza de trabajo. Por un lado, las cifras pueden estar ocultando un volumen de teletrabajo mayor del que ofrecen las estimaciones, ya que el teletrabajo ha sido adoptado en muchos casos como estrategia de autoempleo y como estrategia de flexibilidad de la mano de obra, externalizando trabajadores. Por otro lado, y paradójicamente, podrían estar contándose como

2 Ministerio de Administraciones Públicas (2006): Manual para la implantación de programas piloto de teletrabajo en la Administración General del Estado. MAP, Madrid. 
teletrabajadores actividades que se realizaban anteriormente a distancia sólo que ahora se utilizan ciertas tecnologías de transmisión de datos y de información antes inexistentes. Los problemas de contabilidad derivan justamente de los mismos problemas de definición y conceptualización de lo que se considera teletrabajo ${ }^{3}$.

Más recientemente, la Oficina de Estadísticas de la Unión Europea, Eurostat, publicó para 2005 los datos correspondientes a los indicadores de ciencia y tecnología en los países miembros, datos entre los que figura el porcentaje de empresas con empleados que trabajan parte de su tiempo fuera de la empresa y acceden a los sistemas informáticos a distancia. Los datos ofrecidos por el Eurostat reflejan diferentes ritmos en la penetración social de las tecnologías de la información y la comunicación, así como también puede observarse una cierta «brecha digital» como resultado de su desigual distribución y uso social. Los países con puntuaciones más elevadas en diversos indicadores son Suecia, con una gasto en TIC equivalente al 8\% del PIB (España, $5,2 \%$ ), con un $85 \%$ de individuos entre 16 y 74 años que usaron Internet en el último año (España, 48\%), con un porcentaje de acceso a banda ancha en los hogares del $40 \%$ (España, 21\%), un 41\% de empresas que utiliza el e-comercio (España, 4\%) o con un $40 \%$ de empresas con empleados que trabajan parte de su tiempo fuera de la empresa y acceden a los sistemas informáticos de las mismas a distancia (España, 8\%). A Suecia le sigue un grupo de países como Dinamarca, Finlandia, Eslovaquia, Países Bajos, entre los mejor posicionados. España presenta cifras muy desiguales en los diferentes conceptos ya señalados, comparativamente con los países de la UE. Un reflejo de esta posición lo vemos en el siguiente gráfico:

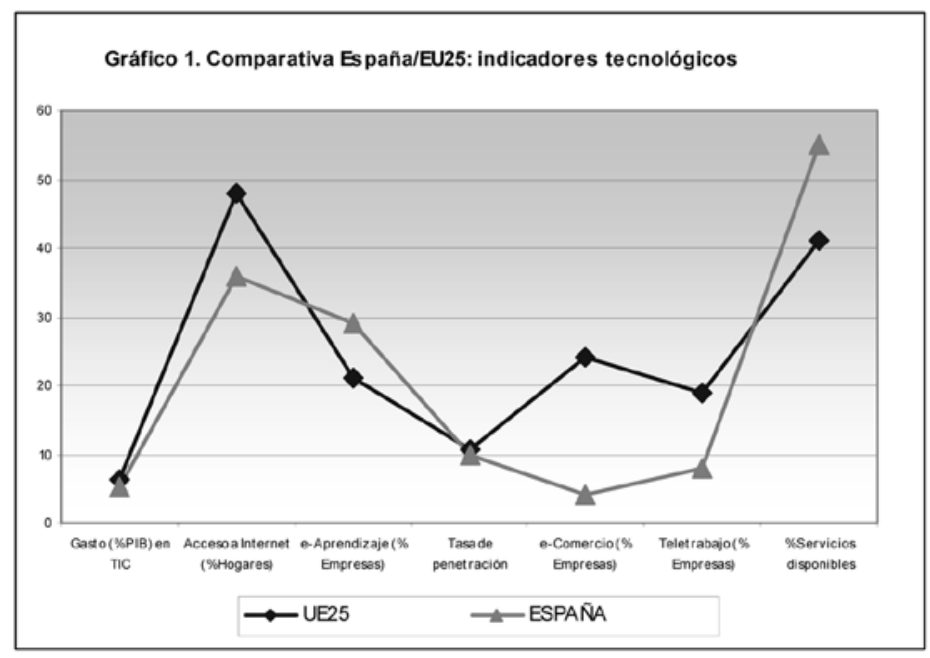

3 Por ejemplo, hay estudios que consideran teletrabajo el efectuado por los trabajadores de los call centres, hecho que haría aumentar considerablemente el número de teletrabajadores en los diferentes países de la Unión Europea y presentaría porcentajes importantes sobre la masa laboral total. 
En la misma tabla exponemos otros indicadores sobre e-Administración:

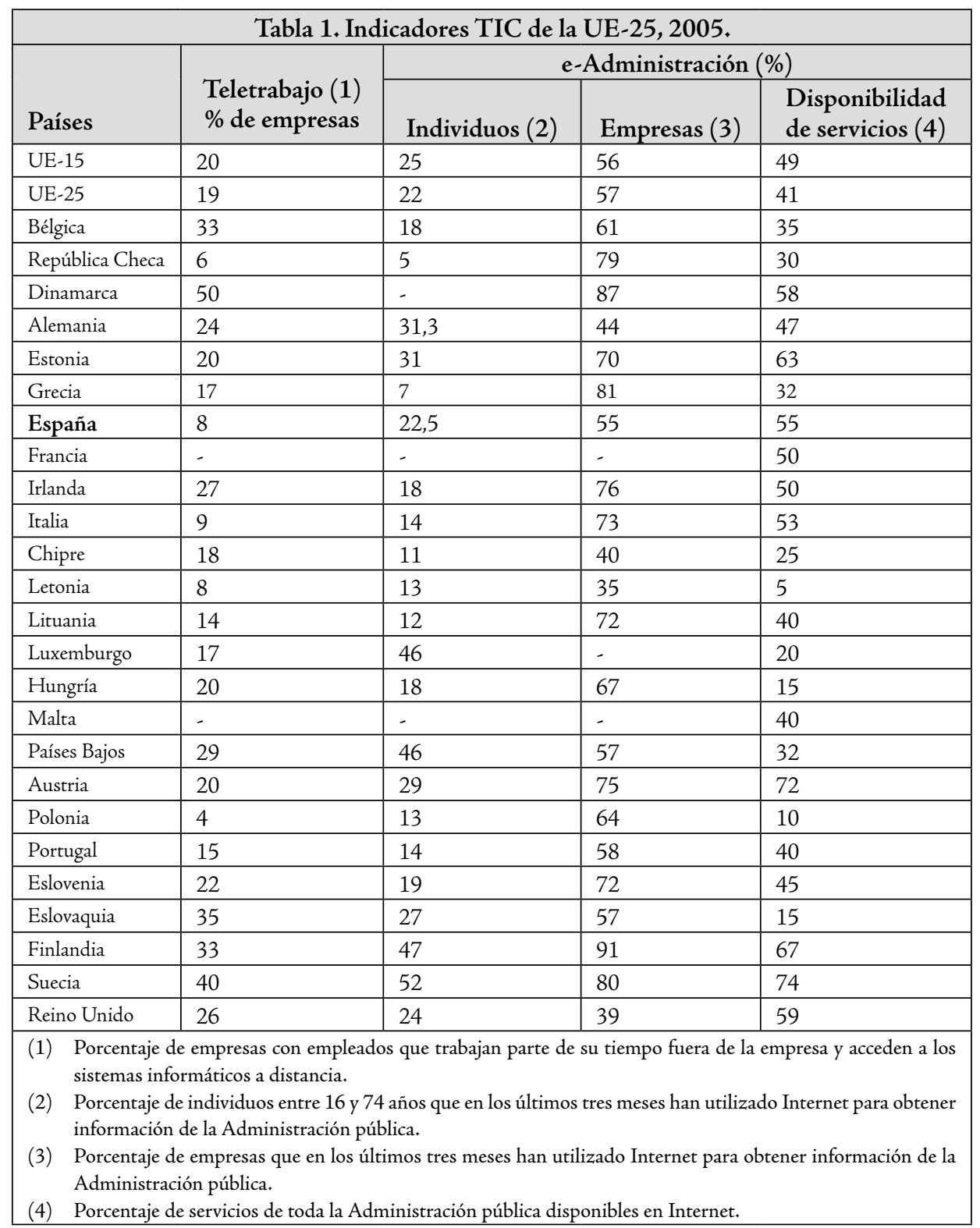

Como se ve en la tabla anterior sólo un $8 \%$ de las empresas españolas practican algún tipo de modalidad de trabajo con sus empleados. Este porcentaje dista mucho de los valores alcanzados por países como Suecia (40\%), Eslovaquia (35\%) o Bélgica (33\%). Sólo Polonia (4\%) y la República Checa (6\%) se encuentran por debajo de 
España en cuanto al uso del teletrabajo por parte de las empresas. Esta cifras reflejan una escasa penetración del teletrabajo en España, al menos del teletrabajo ligado a un mandato empresarial, a once puntos porcentuales de la media de la UE-25.

La implantación del teletrabajo tiene que ver con algunos factores y dimensiones de la penetración de las tecnologías de la información y la comunicación. La denominada brecha tecnológica también se puede observar en los países miembros de la UE, entre aquellos que tienen una mayor difusión en el uso de estas tecnologías y los que se encuentran más rezagados. Así, parece aceptable pensar en la existencia de relación entre el porcentaje de penetración del acceso a la banda ancha y el uso que las empresas hacen del teletrabajo. Cruzando estas dos variables, obtenemos el siguiente gráfico:

Gráfico 2. Tasa de penetración de ancho de banda y empresas que utilizan el teletrabajo

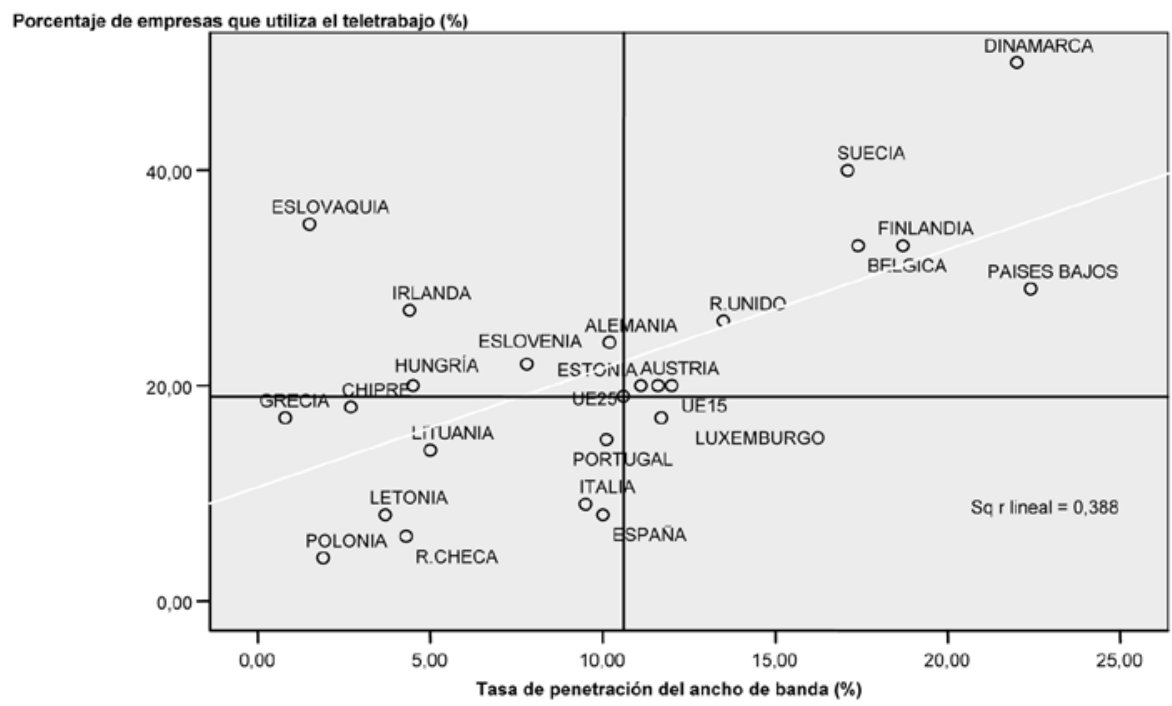

En el gráfico anterior se observa la posición de España respecto al resto de países de la Unión Europea. El cuadrante inferior de la izquierda recoge aquellos países con un escaso desarrollo en la penetración de la banda ancha (en todos los casos por debajo de la media) y, al mismo tiempo, un porcentaje, también por debajo de la media, de empresas que utilizan el teletrabajo. España se sitúa junto a los países de nuevo ingreso en la Unión Europea mientras que los países del norte de Europa destacan en el cuadrante superior derecho con puntuaciones elevadas en las dos variables. Aún así, hay que tener en cuenta que el coeficiente de determinación $r^{2}$ nos ofrece un valor de 0,388 , es decir, el $38 \%$ de la variabilidad observada en el porcentaje de empresas que introduce el tele- 
trabajo, vendría explicado por la variabilidad encontrada en la tasa de penetración del ancho de banda, un porcentaje que podemos considerar moderado-bajo.

\begin{tabular}{|l|l|l|l|l|}
\hline \multicolumn{5}{|c|}{ Tabla 2. Correlaciones entre indicadores TIC en EU, 2005. } \\
\hline & $\begin{array}{c}\text { Gasto en TIC. } \\
\text { Porcentaje del } \\
\text { PIB }\end{array}$ & $\begin{array}{c}\text { Porcentaje } \\
\text { de hogares } \\
\text { con acceso a } \\
\text { Internet }\end{array}$ & $\begin{array}{c}\text { Tasa de } \\
\text { penetración } \\
\text { del ancho } \\
\text { de banda }\end{array}$ & $\begin{array}{c}\text { Porcentaje } \\
\text { de empresas } \\
\text { que utiliza el } \\
\text { teletrabajo }\end{array}$ \\
\hline $\begin{array}{l}\text { Gasto en TIC. Porcentaje del } \\
\text { PIB }\end{array}$ & 1 &, 356 &, 340 &, 196 \\
\hline $\begin{array}{l}\text { Porcentaje de hogares con } \\
\text { acceso a Internet }\end{array}$ &, 356 & 1 &, $788\left(^{* *}\right)$ &, $566\left(^{* *}\right)$ \\
\hline $\begin{array}{l}\text { Tasa de penetración del ancho } \\
\text { de banda }\end{array}$ &, 340 &, $\left.788^{* *}\right)$ & 1 &, $623\left(^{* *}\right)$ \\
\hline $\begin{array}{l}\text { Porcentaje de empresas que } \\
\text { utiliza el teletrabajo }\end{array}$ &, 196 &, $566^{(*)}$ &, $\left.623^{(* *}\right)$ & 1 \\
\hline
\end{tabular}

** La correlación es significativa al nivel 0,01 (bilateral).

Como se ve en la tabla anterior, las mayores correlaciones significativas son las que asocian el porcentaje de hogares con la tasa de penetración del ancho de banda, y esta última con el porcentaje de empresas que utilizan el teletrabajo. Esta última asociación aún siendo substancial, pone de manifiesto que la adopción del teletrabajo por parte de las empresas tiene que ver también con otro tipo de factores más de índole estructural y organizativo, como la estrategia de la empresa, el modelo de gestión, la incorporación de nuevas formas de organización de la producción y del trabajo, entre otros.

Aun asumiendo las prevenciones anteriormente citadas sobre la penetración del teletrabajo en España, y partiendo de la definición más o menos consensuada de teletrabajo del AMET, se pueden identificar sectores productivos y actividades donde el teletrabajo ha tenido una mayor incidencia: generalmente el teletrabajo se ha centrado en actividades profesionales como por ejemplo, la abogacía, el periodismo, el análisis informático, la traducción, las agencias de seguros, la publicidad, las auditorías, las consultorías, la formación o las actividades comerciales, y en sectores productivos en los que se tienden a externalizar ciertas actividades como el diseño de productos, la consultoría, la auditoría, la formación, etc. Un caso especial de teletrabajo cualificado, en relación al contenido de la prestación, es el que tiene que ver con el diseño de productos (tanto industriales como ofimáticos, informáticos, telemáticos...) y que se realiza desde cualquier punto del planeta (en lo que algunos autores, como Romero $(2000)^{4}$, han venido a denominar «teletrabajo transfronterizo»).

4 Romero Burillo (2000). 
Algunos estudios han mostrado una dualización del teletrabajo en España ${ }^{5}$. Por un lado, un teletrabajo centrado en actividades de medio o alto valor añadido a la cadena de valor de los productos y/o servicios. Para este tipo de actividades se requieren teletrabajadores con niveles elevados de cualificación reglada desarrollando tareas que aportan conocimiento e información a las empresas para las que trabajan. Por otro, se ha desarrollado también un tipo de teletrabajo altamente rutinizado (en lo que podríamos denominar una nueva «teletaylorización») centrado en actividades repetitivas, fácilmente externalizables, para las que se requiere escasa formación reglada y que soporta las condiciones de trabajo más precarias. Esta dualización es el resultado de una segmentación difusa con dos extremos que van desde las mejores a las peores condiciones de prestación del teletrabajo.

Por consiguiente, podemos afirmar que no existe una clara asignación de las mejores prácticas de teletrabajo a un tipo de actividad productiva concreta, sino que tanto las mejores como las peores prácticas se encuentran repartidas de forma transversal en los diferentes tipos de actividad. Aún así, hay sectores de actividad en los que aparece con mayor frecuencia el teletrabajo a destajo, con largas jornadas, sin o con escasa protección laboral, con regímenes laborales inducidos, sin penetración sindical, etc. Este es el caso, para España, de las industrias del entretenimiento (sobre todo en el ámbito de los dibujos animados) y de los medios de comunicación (a través de la reconversión de los antiguamente denominados free lance). Especialmente en Cataluña, en la industria del entretenimiento y de la creación de películas de dibujos animados ha irrumpido con fuerza el teletrabajo en su modalidad más desregulada y discrecional por parte de las empresas que dan trabajo, teniendo como resultado unas condiciones de trabajo muy alejadas de las recomendadas por el AMET.

Contrariamente a este sector, el sector del diseño industrial de prototipos y de productos para lanzar al mercado, sobre todo en el sector de las telecomunicaciones y del automóvil, ha logrado poner en marcha un tipo de teletrabajo con mayores garantías de cobertura para los propios trabajadores. Al tratarse de un tipo de actividad muy directamente conectada con la calidad de diseño en el sector industrial, estos «nuevos profesionales» gozan de un estatus, en lo que se refiere a sus condiciones de trabajo, muy similar e incluso superior a los trabajadores presenciales de las empresas de los sectores señalados.

El sector informático de producción del software que elabora productos para el consumo masivo de entretenimiento y de aplicación específica en organizaciones presenta una doble cara. Por un lado, se desarrollan actividades de alto valor añadido realizadas por profesionales que tienen capacidad de gestión y de negociación de sus condiciones de trabajo, pero, por otro, son necesarios programadores neotaylorizados que realizan

5 Belzunegui (2002), Purcalla y Belzunegui (2004), Thibault (2000). 
programas informáticos a destajo similarmente al antiguo mecanografiado que se remuneraba por tecla pulsada ${ }^{6}$.

Probablemente sea el sector de la teleoperación donde se utilizan teletrabajadores para el marketing telefónico de productos de servicios como, por ejemplo, las entidades bancarias, telefonía, energía e industria editorial, el sector que más claramente ha apostado por un tipo de teletrabajo con grandes dosis de control taylorista claramente definido. La existencia de rutinas de actuación muy marcadas por los analistas del trabajo, nos permiten englobar a este tipo de actividades en lo que anteriormente hemos denominado «teletaylorización».

El teletrabajo que se desarrolla bajo los preceptos de la organización taylorista del trabajo, parte de las ideas base que señala Coriat (1993), como a) el desarrollo y la utilización de los instrumentos tecnológicos con el objetivo de minimizar los fallos humanos; b) la separación entre el trabajo de planificación y el trabajo de ejecución; c) la intensificación de los controles sobre el trabajo que, en estos casos, suelen realizarse a través de las tecnologías de la información y la comunicación; d) la concepción economicista de la motivación, y e) un sistema de organización del trabajo altamente jerarquizado. En definitiva, la idea básica es que quien domina el proceso de producción se adueña también de los tiempos de producción. En este sentido, una de las ideas atractivas del teletrabajo, a saber la posibilidad de gestionar más autónomamente los tiempos de trabajo, queda reducida a una mera ilusión en la medida en que este tipo de teletrabajadores al no ejercer control alguno sobre el proceso de producción, pierden también la posibilidad de ajustar el trabajo efectivo a la medida de los tiempos que más les convengan. Este hecho pone de manifiesto, una vez más, cómo es de importante el tiempo como medida de la productividad de la prestación realizada por los (tele) trabajadores en determinadas actividades productivas; en el caso del mecanografiado electrónico, del dibujo animado o de la industria de la programación en serie, el destajo es la norma de productividad, una norma individualizada que imposibilita el diseño personalizado tanto de la carga de trabajo como del tiempo de realización del mismo, así como del ajuste de ambos en una solución autónoma y particularizada. Para este tipo de teletrabajo, la conciliación de la vida laboral y personal puede llegar a ser un objetivo difícilmente alcanzable debido a los ritmos y a las exigencias del trabajo.

De todas formas es imposible, para el caso español, dar cifras sobre la distribución del teletrabajo en estas actividades ya que, como hemos dicho antes, no existe una fuente estadística fiable que permita distribuir pesos porcentuales según las diferentes actividades. La información que se va acumulando hasta el momento procede de estudios de carácter cualitativo, estudios que van arrojando luz sobre un fenómeno, el del teletrabajo, sobre el que es necesario profundizar en el alcance real de sus prácticas.

6 Lara Rodríguez (2003). 
A falta de datos fiables sobre la incidencia del teletrabajo en España en relación a la fuerza de trabajo, y en base a las escasas publicaciones sobre el tema en los últimos años, podría decirse que existe una tendencia de estancamiento en relación al conjunto de la masa laboral. Lejos de crecer exponencialmente el teletrabajo en España, teniendo en cuenta las previsiones más optimistas realizadas a finales de la década de los años noventa del siglo $\mathrm{xx}$, se habría producido un crecimiento muy limitado debido, precisa y paradójicamente, a la flexibilidad del mercado contractual en nuestro país. La liberalización de las formas contractuales ha hecho que los empresarios puedan contratar mano de obra en el lugar clásico de trabajo sin tener que recurrir a una externalización forzosa de ciertas tares o actividades productivas. Se puede afirmar, como ya hemos señalado previamente, que hay una mayor incidencia del teletrabajo en las tradicionales profesiones liberales relacionadas con la transmisión de información y el entretenimiento, pero también con ciertas actividades de sectores industriales centradas en el diseño de los productos y con actividades que pertenecen a lo que Mintzberg (1988) denominó como staff de apoyo.

\section{La regulación del teletrabajo tras el AMET}

Previamente al AMET del 2002 podemos encontrar en España una muy escasa regulación sobre el teletrabajo; la que encontramos se desarrolla en el marco de los convenios colectivos y se centra en algunos aspectos relacionados con el teletrabajo, pero nunca de forma global con el mismo. Así, en ciertos convenios, como por ejemplo, el del Marketing telefónico, el de Siemens Nixdorf España, Ibermática España o DHL Internacional en España, se pueden observar cláusulas que circunscriben la prestación laboral fuera de las oficinas centrales de las empresas, pero en ningún caso se especifica que se esté tratando de teletrabajo. Estas cláusulas se ciñen al uso del correo electrónico, a ciertas condiciones laborales de la prestación en lugares remotos, como por ejemplo tiempo de actuación, salida o reportes, etc., pero nunca claramente o globalmente sobre teletrabajo. Es así porque los convenios o acuerdos de empresa regulan la prestación de trabajadores que aunque siendo teletrabajadores de facto son considerados por las empresas como empleaos de plantilla en el sentido tradicional.

No existe tampoco en España un cronograma de implantación del teletrabajo en diferentes sectores y actividades productivas. En el caso de España se puede afirmar que los acuerdos colectivos que incluyen algún tipo de cláusulas específicas sobre teletrabajo (aunque no se explicite el nombre como tal) no han paliado la falta de regulación genérica ni específica sobre el mismo. Sin embargo los sindicatos han presionado para ir incorporando en los contenidos de la negociación colectiva, sobre todo de convenios 
colectivos de empresa, algunas cláusulas que regulan ciertas peculiaridades que se desarrollan alrededor del teletrabajo. Como ejemplo, el Acuerdo Interconfederal para la Negociación Colectiva 2003 incorporó los preceptos del AMET, formando parte de las orientaciones dirigidas a los negociadores para el diálogo social.

El hecho de que no exista una regulación general que acoja las relaciones contractuales entre empresas y teletrabajadores, no quiere decir que sea automáticamente sustituida por una regulación específica en base a acuerdos y/o convenios colectivos. Los escasos convenios de empresa en los que se ha recogido algún aspecto referente al teletrabajo presentan importantes vacíos en cuanto a la regulación de las condiciones reales de ejercicio del teletrabajo; además hay que tener en cuenta que en el caso español los contenidos de negociación son más bien reducidos y que bajo el epígrafe genérico de «organización del trabajo» quedan incluidos todos aquellos posibles contenidos que la dirección de las empresas se reserva y no son sujetos de negociación.

Las centrales sindicales con mayor representación en España, CCOO y UGT, se han visto, esta última década, concernidas por el desarrollo del teletrabajo en diversas empresas y sectores económicos. En la medida en que las instituciones regionales y municipales han ido fomentando la puesta en práctica de experiencias de teletrabajo (sobre todo en la modalidad de centros de recursos compartidos o telecentros), los sindicatos han ido participando en el diseño de estas propuestas, intentando introducir elementos de protección destinados a los trabajadores que se han visto involucrados. Las instituciones municipales y regionales han sido las más entusiastas a la hora de poner en marcha experiencias de teletrabajo como forma de autoempleo y de corrección del problema de la desocupación en España; algunas de estas iniciativas han tenido éxito en zonas rurales, pero en la mayoría de los casos, estas iniciativas han sido reconvertidas a experiencias de utilización de las tecnologías de la información y la comunicación, no estrictamente a lo que aquí se viene considerando como teletrabajo.

Por tanto, podemos afirmar que, en los últimos diez años, no ha habido cambios regulatorios de carácter general y transversal que afecten a las partes contractuales ni a la Administración; del mismo modo, tampoco se ha observado regulación autonómica, regional y/o local para paliar la falta de regulación. Desde nuestro punto de vista, la causa de esta falta de regulación se debe, entre otros factores, al hecho de que el teletrabajo se ha venido considerando como una parte sustantiva de la denominada «organización del trabajo» que, en los convenios colectivos, queda reservada a la parte empresarial y, por consiguiente, es escasamente negociable. Este hecho hace que en lo relativo al teletrabajo haya una fuerte discrecionalidad de la parte empresarial en la delimitación de las prácticas asociadas a las diversas formas de teletrabajo. 
En España la jurisprudencia sobre temas de teletrabajo ha sido prácticamente inexistente a lo largo de los últimos años, recogiendo sólo alguna sentencia con carácter aislado, como por ejemplo:

- Sentencia del Tribunal Superior de Justicia de Cataluña 9178/2004, sobre la presunción de existencia de contrato de trabajo y de dependencia de una teletrabajadora que realizaba tareas de mecanografiado.

- Sentencia del Tribunal Superior de Justicia de Cataluña 25/2003 sobre la obligatoriedad de un trabajador presencial a convertirse en teletrabajador (tras comunicación verbal) como consecuencia de cambios organizativos en la empresa.

- Sentencia del Tribunal Supremo de 11 de abril del 2005, que concluye que la implantación de un sistema de trabajo a domicilio (en este caso de teletrabajo), pasando el trabajador a desarrollar parte de su actividad en su domicilio, en lugar de las oficinas de la empresa, exige la aceptación voluntaria del trabajador, no pudiendo establecerse por acuerdo o convenio colectivo, ni tampoco por modificación de las condiciones de trabajo.

Por su importancia, esta última doctrina jurisprudencial fija los límites a los que tiene que sujetarse una decisión de reorganización empresarial mediante la implantación de un sistema de teletrabajo. El Acuerdo Marco Europeo sobre Teletrabajo asegura el carácter voluntario para el trabajador, pero su falta de incorporación al Derecho Interno, al no haber sido recibido por la negociación colectiva, no impide al Tribunal Supremo reconocer esa misma conclusión.

\section{El papel de los interlocutores sociales y las condiciones de trabajo}

Los sindicatos españoles, considerando la elevada discrecionalidad por parte de la empresa en la implantación de los diseños de teletrabajo, han venido incorporándose al debate sobre las prácticas del teletrabajo demandando una mayor participación de los representantes de los trabajadores en la marcha de dichos diseños. Por ejemplo, se ha puesto una especial atención a todo lo que tiene que ver con la situación contractual de los teletrabajadores, ya que una buena parte de los mismos han aparecido en el mercado como trabajadores autónomos, considerando los sindicatos una más que probada relación de subordinación entre la mayoría de los teletrabajadores y las empresas que los contratan como realidades jurídicas independientes. Para los sindicatos, el teletrabajo que pueda demostrar que en la prestación existe esta subordinación, ha de ser asimilado a las condiciones de salarización previstas en la propia norma (Estatuto de 
los Trabajadores), sin perjuicio del desarrollo de normas de regulación colectivas que puedan acoger a este colectivo de trabajadores.

Algunos ejemplos de la discrecionalidad que envuelve al fenómeno del teletrabajo, como resultado de la falta de criterios regulatorios de los diversos ámbitos que se ven afectados, son los siguientes:

a) Las empresas trabajan con bases de datos de los empleados confidenciales que no son facilitadas a sus representantes laborales. Generalmente tampoco las empresas han adoptado una política de facilitar las direcciones electrónicas de estos teletrabajadores a los comités de empresa o a sus representantes laborales, con lo cual se dificulta el contacto entre aquéllos y el resto de trabajadores.

b) Por otra parte, las empresas trabajan con protocolos internos de confidencialidad en el manejo y transmisión de datos relativos a la prestación de los teletrabajadores.

c) Para solventar las deficiencias de formación de los teletrabajadores las empresas más avanzadas han puesto a disposición de sus empleados (teletrabajadores o no) aulas virtuales en su Intranet a través de las cuales los trabajadores pueden acceder a algún tipo de formación requerida para el puesto de trabajo. La formación, así, sale del ámbito de la negociación colectiva y de las horas de trabajo, para pasar a ser una actividad que relaciona individualmente al teletrabajador con la empresa.

d) Los teletrabajadores en domicilio con estatus de autónomos no tienen la protección en materia de seguridad y salud como el resto de trabajadores, aunque en casos puntuales las empresas realizan inspecciones (siempre con el permiso del teletrabajador) de las instalaciones desde las que opera. Los teletrabajadores que practican el mobility y están ligados contractualmente a la empresa, si gozan de la protección de seguridad y salud que brindan las empresas, además de negociar ciertas especificidades como los riesgos asociados a la movilidad con la que realizan la prestación. En casos puntuales, la empresa también vela porque las condiciones de salud e higiene de las empresas clientes sean las adecuadas para realizar la prestación.

e) No existe una jornada laboral estándar en las prácticas del teletrabajo, variando enormemente la flexibilidad/rigidez de la misma en función de otros factores como, por ejemplo, el estilo de dirección de la empresa, la actividad, los clientes de la misma, el tipo de mano de obra, la carga de trabajo, etc. Sin embargo, puede afirmarse, en líneas generales, que el teletrabajo no ha supuesto en la mayoría de los casos una relajación de la prestación, si se entiende por aquélla, un tipo de prestación más conciliadora con otros ritmos de vida marcados por las obligaciones diarias. Bien al contrario, en la mayoría de estudios se pone de manifiesto la existencia de una mayor carga de trabajo trasladada hacia el teletrabajador que acaba repercutiendo en el alargamiento de las jornadas laborales diarias. 
Los sindicatos consideran, asimismo, que con el teletrabajo se dificulta la conexión de los representantes sindicales con sus representados, así como de éstos con el propio sindicato. Para corregir este déficit de comunicación ante el desarrollo unilateral del teletrabajo por parte de las empresas, los sindicatos se han comenzado a plantear dos necesidades de futuro imprescindibles: por un lado potenciar los comités de empresa europeos ya que una buena parte del teletrabajo puede desarrollarse en la modalidad de trabajo transfronterizo, y por otro, garantizar la interlocución para los teletrabajadores en los convenios colectivos de empresa. En cualquier caso, los sindicatos españoles mayoritarios están viendo la necesidad de crear redes de comunicación telemáticas con sus representantes y con los teletrabajadores, utilizando como vehículo de comunicación las tecnologías de la información y de la comunicación. En este terreno se plantea un conflicto de importancia creciente ya que los sindicatos consideran que estos instrumentos han de ser los mismos que la empresa pone a su alcance para realizar la prestación, mientras que las empresas, por lo general, se niegan a que sean utilizados como instrumento de comunicación sindical. Existen algunos casos en los que la filial de una empresa en un país garantiza a los representantes de los trabajadores la utilización de bases de datos y correos electrónicos de los trabajadores para que puedan contactar con ellos, mientras que otra filial en otro país lo prohíbe sin ninguna posibilidad de negociación.

Las organizaciones patronales y las empresas que han implantado el teletrabajo, tienen la tendencia a considerar el teletrabajo en el marco de la relación contractual individual entre la organización y el trabajador; por consiguiente, lo concerniente a las condiciones de trabajo de los teletrabajadores tendrían que resolverse en el plano de la relación individual de trabajo.

En conclusión, en el caso de España se puede afirmar que el Acuerdo Marco Europeo sobre Teletrabajo no ha llevado a los interlocutores a adoptar líneas de actuación consensuadas para la regulación del teletrabajo, a pesar de que haya habido una cierta voluntad de compromiso para acercar las recomendaciones incluidas en el AMET al diálogo social. Por su parte, los sindicatos han presionado para ir incorporando en los contenidos de la negociación colectiva, sobre todo de convenios colectivos de empresa, algunas cláusulas que regulan ciertas especificidades y peculiaridades que se desarrollan alrededor del teletrabajo, como descansos, tiempos de trabajo efectivo, equipamiento y gastos de conexiones domiciliarias.

\section{Teletrabajo y Administración pública en España}

Los antecedentes de la incorporación del teletrabajo en la Administración pública española deben situarse en preocupaciones paralelas como, por ejemplo, la conciliación 
de la vida profesional y personal de los trabajadores. Así, la Declaración para el diálogo social en las Administraciones públicas, firmada por la Administración y las centrales sindicales UGT, CCOO y CSI-CSIF el 21 de septiembre de 2004, estableció entre sus objetivos la mejora de las condiciones de trabajo y la profesionalización de los empleados públicos, como factores que ayuden al aumento de la calidad de los servicios. A partir de esta Declaración, el 4 de marzo de 2005 el Consejo de Ministros aprobó el Plan Concilia que recoge una serie de medidas para hacer posible la conciliación en el ámbito público. Posteriormente, a lo largo de los meses finales de 2005 y el primer trimestre del 2006 el Ministerio de Administraciones Públicas desarrolló en sus servicios centrales un Plan piloto para la aplicación del teletrabajo entre sus empleados para favorecer la conciliación de la vida laboral i personal. La evaluación de este plan puso de manifiesto que los trabajadores mantuvieron e incluso en casos incrementaron la productividad a la vez que su valoración sobre conciliación fue positiva ${ }^{7}$.

Concretamente en España el nuevo Estatuto del Empleado Público abre una puerta a la realización de ciertos tipos de trabajo en la modalidad de teletrabajo con el objetivo central de poder conciliar mejor la vida laboral y la profesional. Sin embargo, como muchas de las cuestiones que giran en torno al teletrabajo, la mención a éste en el nuevo Estatuto es vaga, sin directrices claras ni objetivos específicos que supongan un determinado grado de alcance de su aplicación. Hay que tener en cuenta que las líneas de actuación de dicha reforma, cuyo objetivo fundamental es la modernización de la Administración pública, son las siguientes: a) la flexibilidad: redacción de una Ley de Agencias. Funcionarán a partir de contratos de gestión por objetivos, y dispondrán de capacidad de decisión sobre los recursos asignados; $b$ ) una nueva cultura de evaluación de resultados: Agencia de Evaluación de la Calidad de los Servicios. Realizará auditorias de calidad de los servicios que se prestan desde la Administración; $c$ ) un nuevo modelo de empleo público: 1) Estatuto de la Función Pública. Con un nuevo sistema de retribución que tendrá en cuenta la evaluación objetiva del desempeño del puesto de trabajo y con mejores condiciones laborales; 2) Reforma del proceso de selección. Nuevo sistema de acceso, más ágil, que tenga en cuenta, en mucha mayor medida, la importancia de los períodos de formación y prácticas; 3) Integración de los discapacitados. Se hará efectiva la reserva legal del $5 \%$ de las plazas para personas discapacitadas, que hasta ahora no se ha cumplido; 4) Conciliación de la vida laboral y familiar. Fórmulas de adaptación del teletrabajo a la Administración, así como la adopción de una mayor flexibilidad en los horarios de trabajo; d) aprobación de un Código ético del empleado público, dirigido a fomentar la vocación de servicio público; e) nuevo escenario de relaciones laborales: Foro del Diálogo social. Comunicación directa con los representantes de los empleados públicos, como pauta normal para la toma de de-

7 Ministerio de Administraciones Públicas (2006), Libro electrónico sobre el Plan concilia: horarios y teletrabajo. 
terminadas decisiones que afectan a este colectivo; $f$ ) modernización tecnológica: Plan Conecta. Proyectos como la Administración General del Estado en Red o el DNI electrónico; $g$ ) impulso a la Administración periférica del Estado. Renovación de las instalaciones y los recursos. Propuestas de modificaciones, tanto normativas como de gestión ordinaria de los servicios, que se están llevando ya a la práctica.

El desarrollo del teletrabajo como modalidad de prestación en la Administración pública puede encontrarse con los mismos problemas que su expansión en el ámbito de las empresas privadas. En primer lugar puede darse el caso de que la aplicación esté exclusivamente en manos de los responsables del servicio concreto de la Administración y no dentro de un programa de acción más amplio, de una planificación a medio y largo plazo que consiga los dos objetivos propuestos inicialmente: modernización de la Administración y conciliación de vida laboral y vida personal. En cierta manera se trataría de una cierta discrecionalidad en cuanto a la incorporación de esta modalidad de prestación que estaría en función más de la disposición de los responsables que no de un estudio pormenorizado de los servicios y actividades que pueden acoger dicha modalidad de trabajo. Este hecho ha venido tratándose en los planes piloto puestos en marcha por el Ministerio de Administraciones Públicas, atendiendo a una de las recomendaciones efectuadas en el Manual del Teletrabajo elaborado por el mismo Ministerio. Esta recomendación se centra en el proceso dialogado de la puesta en marcha del teletrabajo, donde necesariamente deberán participar cuadros dirigentes, potenciales teletrabajadores y representantes sindicales de los mismos. La experiencia de los planes piloto demuestra que se puede lograr consensuar la implementación del teletrabajo en determinados servicios de la Administración, pero no garantizan que la futura extensión del teletrabajo se haga en las mismas condiciones.

En segundo lugar, falta una evaluación de qué tipo de servicios puedan prestarse en modalidad de teletrabajo sin menoscabo de la eficacia y eficiencia en su prestación. Como se sabe, entre los objetivos de modernización de la Administración están lograr la mayor eficacia y eficiencia en la relación con los ciudadanos usuarios de dichos servicios. La pregunta pertinente aquí es si dicha eficacia y eficiencia puede verse afectada al incorporar la modalidad del teletrabajo. En términos de productividad, las primeras evaluaciones realizadas de los planes piloto, muestran que aquella, lejos de disminuir o estancarse, ha aumentado en determinadas circunstancias.

En tercer lugar, el Estatuto del Empleado Público no se pronuncia sobre las condiciones de trabajo de los teletrabajadores de un servicio determinado. Al respecto, la Administración pública hace suyas las recomendaciones que al respecto efectuó en su día el Acuerdo Marco Europeo sobre teletrabajo a la hora de plantear los planes piloto y de realizar su seguimiento. Sin embargo, la discrecionalidad a la hora de su implementación puede acarrear también desajustes o problemas respecto a las condi- 
ciones en que se ha de prestar el servicio. Entre las características de la prestación en modalidad de teletrabajo está, según siempre el Acuerdo Marco, la de la posibilidad de reversibilidad a la situación anterior a la del teletrabajo en el caso en el que el trabajador así lo demande. Esta casuística puede presentar problemas cuando se ha decidido que un servicio en su totalidad se preste en modalidad de teletrabajo.

Para tratar de abordar estos y otros interrogantes en relación a la adopción del teletrabajo en el marco de los servicios de la Administración General del Estado, el Ministerio de Administraciones Públicas elaboró un manual para tratar de encauzar la puesta en práctica de las experiencias de teletrabajo en el seno de la propia Administración General del Estado y, de esta manera, reducir el margen de discrecionalidad en la implementación.

No hay que perder de vista que el objetivo fundacional del Ministerio de Administraciones Públicas en su apuesta por el teletrabajo es lograr la conciliación de la vida laboral y personal de los trabajadores de dicha Administración. Así, queda reflejado como declaración de intenciones en el manual: «Los Programas de teletrabajo se enmarcan en los objetivos del Plan Concilia, por lo que el fin primordial de cada Programa es la conciliación de la vida personal y familiar a través de la flexibilidad para realizar el trabajo desde el domicilio y en el horario que se pacte con la empleada o con el empleado, todo ello sin disminución de la cantidad y calidad del servicio». Paralelamente, el Ministerio pretende potenciar el trabajo basado en la denominada dirección por objetivos y no medido en tiempo de presencia en el centro de trabajo, así como aumentar el compromiso con la calidad del trabajo y reducir el absentismo laboral.

Dicho manual se reduce básicamente a una serie de indicaciones acerca de cómo ha de ser el procedimiento o procedimientos para poner en marcha el plan piloto: a quién va dirigido el teletrabajo, selección de los candidatos a teletrabajar, estructura e infraestructura de la prestación, etc. El manual no llega a anticipar cómo han de ser las condiciones de trabajo aunque de forma implícita se sobreentiende que han de ser las mismas que la de los trabajadores presenciales, y nunca en menoscabo de los derechos adquiridos.

Por su parte, el Consejo Económico y Social de España elaboró un Dictamen sobre el Proyecto de Real Decreto por el que se regula el teletrabajo en la Administración General del Estado, con fecha 21 de marzo de 2007. En este Proyecto de Real Decreto se especifican, vagamente también, algunas de las condiciones en las que se ha de desarrollar la práctica del teletrabajo, en lo que respecta a la prestación de los nuevos teletrabajadores: el carácter voluntario del teletrabajo, así como la autorización por los órganos correspondientes para adquirir dicha condición. También recoge otros aspectos como que la parte de la jornada laboral que se realice bajo forma de teletrabajo no podrá exceder el $40 \%$, o que la retribución de los teletrabajadores no ha de ser inferior 
a la que percibían antes de acogerse a dicha modalidad. Los sucesivos artículos del Proyecto de Real Decreto dedican su espacio a aspectos organizativos y de estructuras para la prestación, a la evaluación del trabajo, a las garantías de protección y de confidencialidad de los datos y a la seguridad y salud en el trabajo.

Sin embargo, el informe del Consejo Económico y Social advierte que ya existen diversas normas que recogen los derechos de participación y negociación colectivas de los empleados públicos, normas en cuyo contexto debería implantarse la regulación de los aspectos propios de la prestación en formato de teletrabajo. Por ejemplo, respecto a los funcionarios públicos, la Ley 9/1987 de Órganos de representación, determinación de las condiciones de trabajo y participación del personal al servicio de las Administraciones públicas, establece que deben ser objeto de negociación en su ámbito respectivo las materias que afecten a las condiciones de trabajo y al ámbito de relaciones de los funcionarios públicos con sus organizaciones sindicales. Para los trabajadores con el estatus de personal laboral, el CES indica que el marco a considerar debería ser el II Convenio Único para el personal laboral de la Administración General del Estado (resolución del 20 de octubre de 2006).

En el documento emitido por el CES se considera tarea central la determinación de los sistemas de evaluación del desempeño del trabajo en modalidad de teletrabajo. $\mathrm{Al}$ respecto, el dictamen advierte que la evaluación del rendimiento o del desempeño de los puestos de trabajo es un aspecto escasamente desarrollado al estar pendiente de la negociación de los sistemas de evaluación.

En resumen, podemos concluir tras la lectura de los documentos elaborados por diferentes instancias, que la implementación del teletrabajo en la Administración General del Estado se encuentra en un momento que podríamos llamar experimental. Los agentes sociales están a la expectativa de las primeras valoraciones sobre los planes piloto y sobre la concreción de aspectos centrales como los mencionados anteriormente, en especial la evaluación del rendimiento y del desempeño como factores decisivos en la carrera profesional. Se trata de que el documento final que dará luz al Real Decreto sea un documento que especifique sistemas objetivos y transparentes pactados con las organizaciones sindicales representativas en el conjunto de las Administraciones públicas.

\section{Bibliografía}

Belzunegui, A. (2002) El teletrabajo: estrategias de flexibilidad. Madrid: Consejo Económico y Social. 
Belzunegui, A. y Moreno, J. (comp.) Coperació tecnològica i Mediterrània. Barcelona: Consorci Universitari del Baix Penedès.

Consejo Económico y Social (2007) Dictamen sobre el Proyecto de Real Decreto por el que se regula el teletrabajo en la Administración General del Estado. Madrid: CES.

Carrasquer, P.; Borras, V;; Torns, T. (2004) «La conciliación de la vida familiar y laboral», Revista Sociología del Trabajo, nº ${ }^{\circ}$ 50: 111-138.

Coriat, B. (1993) El taller y el robot. Madrid: Editorial Siglo XXI.

EuroCommerce and UNI-Europa: European Agreement on Guidelines on Telework in Commerce. http://www.union-network.org.

Lara Rodríguez, A. (2003) «El telemarketing en España: materiales para una cartografía del mundo del trabajo contemporáneo», Revista Sociología del Trabajo, $\mathrm{n}^{\circ} .49: 27-59$.

Ministerio de Administraciones Públicas (2006) Manual para la implantación de programas piloto de teletrabajo en la Administración General del Estado. Madrid: MAP.

Ministerio de Administraciones Públicas (2006) Libro electrónico sobre el Plan concilia: horarios y teletrabajo. Madrid: MAP.

Mintzberg. H. (1988) La estructuración de las organizaciones. Barcelona: Editorial Ariel.

Purcalla, M.A. y Belzunegui, A. (2004) «Marcos jurídicos y experiencias prácticas de teletrabajo», Revista Aranzadi Social, no 18, Febrero.

Romero Burillo, A. (2000) «El teletrabajo: algunas consideraciones jurídicolaborales». En A. Belzunegui y J. Moreno (comp.) Coperació tecnològica i Mediterrània. Barcelona: Consorci Universitari del Baix Penedès.

SÁez Soro, E. (2004) «Transformaciones en la figura del profesional a través del teletrabajo», Revista Sociología del Trabajo, nº. 51:61-82.

Thibault, J. (2000) El Teletrabajo. Madrid: Consejo Económico y Social. 